\title{
Mesoporous Silica for Triphase Nucleophilic Substitution Reactions
}

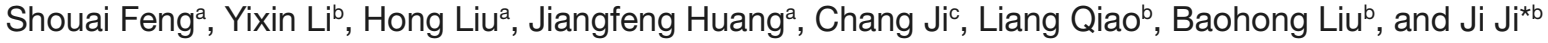

\begin{abstract}
Mesoporous silica SBA-15 is shown to be a very efficient alternative to phase transfer catalyst salts for two-phase nucleophilic substitutions. The two-phase reaction can efficiently take place in the absence of PTCs. The high catalytic activity and reaction rates can be attributed to the amphiphilic and negatively charged surface of SBA-15 as well as the rapid ingress of the reactants and egress of the products.
\end{abstract}

Keywords: Nanoreactor · Nucleophilic substitutions · Phase transfer catalyst

\section{Introduction}

Phase transfer catalysis in two-phase systems is an elegant method for a wide range of organic reactions ${ }^{[1,2]}$ including nucleophilic substitutions. ${ }^{[3]}$ Phase transfer catalysts (PTCs) are generally quaternary ammonium salts, which might be immobilized on a polymeric matrix ${ }^{[4]}$ to be recycled for cost effective and environmental purposes. More recently, ketiminium salts[5] have also been applied as PTCs for asymmetric synthesis. Although effective PTCs can successfully facilitate the reaction between water-insoluble reactants in the organic phase and hydrophilic ionic species in the aqueous phase, many of these catalysts are hazardous. Moreover, the use of harmful solvents such as toluene or methylene chloride involved in conventional two-phase reactions ${ }^{[6]}$ is not environmentally friendly and novel green methods are desired. ${ }^{[7,8]}$ Eckert et al. ${ }^{[9]}$ have demonstrated that room temperature ionic liquids can be used as alternative catalytic phases for cyanide displacement of benzyl chloride.

${ }^{*}$ Correspondence: Dr. J. Ji ${ }^{\mathrm{b}}$

E-mail: jiji@fudan.edu.cn

aTechnology Centre of China Tobacco Guangxi Industrial Co., LTD, Guangxi Province 530001, China

bDepartment of chemistry, Fudan University, Shanghai 200433, China

'Department of Chemistry and Biochemistry,

Texas State University-San Marcos, 601 University

Drive, San Marcos, Texas 78666, USA
Besides PTCs, other heterogeneous catalytic strategies have been developed with various nanoparticles or nanoporous materials such as zeolites and mesoporous silica molecular sieves as matrices. Mesoporous silica materials have recently attracted lots of interest in many catalytic reactions including acid/base catalysis, hydrogenation, desulfuration, oxidation, asymmetric synthesis and enzyme catalysis. ${ }^{[10]}$ The surface of the mesoporous silica can be grafted with different groups, which can greatly modify the surface functionality and textural properties, ${ }^{[11]}$ and consequently affect the corresponding catalytic behavior. Since the amphiphilic surface of the mesoporous silica is a suitable host for both hydrophilic and hydrophobic molecules, the concept of liquid-liquid-solid 'triphasic catalysis' has been investigated. ${ }^{[12]}$ Furthermore, the submicrometer channels in silica offer advantages such as easy accessibility, rapid diffusion, and favorable mass transfer for substrates/products into and out of the mesopores. ${ }^{[13]}$ Shiju and co-workers ${ }^{[14]}$ combined acidic and basic functions on periodic mesoporous silica to obtain an efficient and robust bifunctional catalyst for two tandem reactions. Lectka and his colleagues $^{[15]}$ reported a mechanistically based study of homogeneous bifunctional catalyst systems for the asymmetric synthesis of $\beta$-lactams in high chemical yield, diastereoselectivity, and enantioselectivity.

Here, we demonstrate the use of ordered mesoporous silica (SBA-15) without any modification as a low-cost and powerful nanoreactor for liquid-liquid nucleophilic substitution reactions. The twophase reaction can efficiently take place in the absence of PTCs (Fig. 1). The high catalytic activity and reaction rates can be attributed to the amphiphilic and negatively charged surface of SBA-15 as well as the rapid ingress of the reactants and egress of the products.

\section{Experimental}

Reagents, Standards and Samples

Tetramethoxysilane (TMOS), EO20PO70EO20 [denoted P123, where $\mathrm{EO}$ is poly(ethylene oxide) and PO isopoly(propylene oxide)], aluminum isopropoxide, ammonium bicarbonate, cetyltrimethylammonium bromide (CTAB), 3-aminopropyl, 3-aminopropyltrimethoxysilane (APTMS), methyltriethoxysilane (MTES) were purchased from Aldrich. All reagents were used as received without further purification. Deionized water (18.4 $\mathrm{MWcm}$ ) used for all experiments was obtained from a Milli-Q system (Millipore, Bedford, MA,USA).

\section{Synthesis of Nanomaterials Mesoporous silica}

SBA-15 was prepared by using triblock copolymer Pluronic P123 (EO20-PO70EO20) as a structure directing agent under acidic condition according to the literatures. ${ }^{[16]}$ For the same batch, hydrothermal treatments with different temperatures and times resulted in various pore sizes. MCM41 was prepared by using cationic surfactant cetyltrimethylammonium bromide (CTAB) as a structure-directing agent under basic conditions. The calcinations were carried out at $550{ }^{\circ} \mathrm{C}$ in air for $5 \mathrm{~h}$ to fully remove the template from the mesoporous silica.

\section{Surface Modification}

3-Aminopropyl functionalized SBA15 (denoted as SBA-15- $\mathrm{NH}_{2}$ ) was obtained via a refluxing process of 0.221 g (1.0 mmol) of 3-aminopropyltrimethoxysilane (APTMS) and $1.0 \mathrm{~g}$ of SBA15 with pore size of $12 \mathrm{~nm}$ in dried toluene at $120{ }^{\circ} \mathrm{C}$ for $12 \mathrm{~h}$. Methyl modified SBA-15 (denoted as SBA-15- $\mathrm{CH}_{3}$ ) was prepared via a refluxing process of $0.1 \mathrm{~g}$ of methyltriethoxysilane (MTES) and 1.0 $\mathrm{g}$ of SBA-15 with pore size of $12 \mathrm{~nm}$ in toluene at $120^{\circ} \mathrm{C}$ for $12 \mathrm{~h}$. 


\section{Characterization}

The TEM images were recorded by a JEOL 2011 microscope operated at 200 $\mathrm{kV}$. A zeta $(\zeta)$ potential meter (Malvern Zetasizer Nano) was used to measure the zeta potentials at $298 \mathrm{~K}$ in water. X-ray diffraction (XRD) patterns were recorded on a German Bruker D8 advanced X-ray diffractometer with Ni-filtered $\mathrm{Cu} \mathrm{K \alpha}$ radiation (wavelength of $0.154 \mathrm{~nm}$ ) at a voltage of $40 \mathrm{mV}$ and a current of $40 \mathrm{~mA}$. The powdery samples were pressed and loaded onto the sample plate. The XRD patterns were recorded and analyzed by the Bruker software DIFFRAC-XRD Commander.

\section{Nucleophilic Displacement \\ Reactions}

A $1.0 \mathrm{~mL}$ of aqueous solution containing $10.0 \mathrm{mmol}$ of $\mathrm{NaOH}$ and $7.5 \mathrm{mmol}$ of phenol or $p$-cresol was added to $5 \mathrm{mmol}$ of benzyl halide with vigorous stirring. For SBA-15 catalyzed reaction, $100 \mathrm{~mL}$ of SBA- 15 solution $(20 \mathrm{mg} / \mathrm{mL}$, dispersed in water) was also added into the mixture described above. For traditional PTC catalyzed reaction, $0.15 \mathrm{mmol}$ of $\mathrm{TBABr}$ was added instead of SBA-15. After the reaction was completed, the mixture was extracted with diethyl ether and filtered (if SBA-15 is used). The combined organic phases were dried over anhydrous $\mathrm{MgSO}_{4}$ followed by filtration. The solvent was removed under vacuum and the residue was dissolved in $\mathrm{CH}_{3} \mathrm{CN}$ and analyzed by HPLC (Agilent 1100). For HPLC analysis, the separation was carried out on a HP Stable Bond-C18 $(4.6 \mathrm{~mm} \times 250 \mathrm{~mm}, 5$ $\mu \mathrm{m})$ column using a mobile phase of $15 \%$ water and $85 \% \mathrm{CH}_{3} \mathrm{CN}$ at a flow rate of $0.8 \mathrm{~mL} / \mathrm{min}$

\section{Results and Discussion}

SBA-15 mesoporous silica materials (as shown in Fig. 1, Fig. 2a) were prepared according to the literature. ${ }^{[16]}$ The pore diameter is about $12 \mathrm{~nm}$. Powder X-ray diffraction (XRD) patterns in Fig. 2c show three diffraction peaks that can be indexed to highly ordered hexagonal mesostructures. Transmission electron microscopy (TEM) images of SBA-15 also show good mesostructural regularity in large domains. The synthesis of benzyl phenyl ether was chosen as a model liquid-liquid nucleophilic substitution to evaluate the catalytic efficiency. The reactions were carried out without any organic solvent. For a typical experiment, $1.0 \mathrm{~mL}$ of aqueous solution containing $10.0 \mathrm{mmol}$ of $\mathrm{NaOH}$ and 7.5 mmol of phenol was added at room temperature to $5 \mathrm{mmol}$ (approximately 0.6 $\mathrm{mL}$ ) of benzyl halide in the presence of various catalysts under vigorous stirring. The reaction products were monitored by
HPLC and the corresponding reaction profiles are shown in Fig. 3.

For a conventional biphasic reaction without catalyst, it was reported that the product conversion would be only $3 \%$ after $3 \mathrm{~h}$ of stirring. ${ }^{[8]} \mathrm{A}$ much higher conversion of $87 \%$ can be obtained within two hours of reaction if TBABr was employed as PTC (Fig. 3). Comparatively, when SBA-15 was used as an interfacial catalyst, an even higher conversion of $93 \%$ was achieved within the same period of time in the absence of any PTC reagent. The difference shown in catalytic efficiency between SBA-15 and $\mathrm{TBABr}$ is also obvious for shorter reaction time (i.e. $1 \mathrm{~h}$ ). Assuming a simple secondorder reaction model, the rate constants for the nucleophilic substitution catalyzed by SBA- 15 and TBABr were found to be 0.17 and $0.07 \% \%^{-1} \mathrm{~min}^{-1}$, respectively, using a linear regression method. The fitted kinetics curves are shown in Fig. 3.

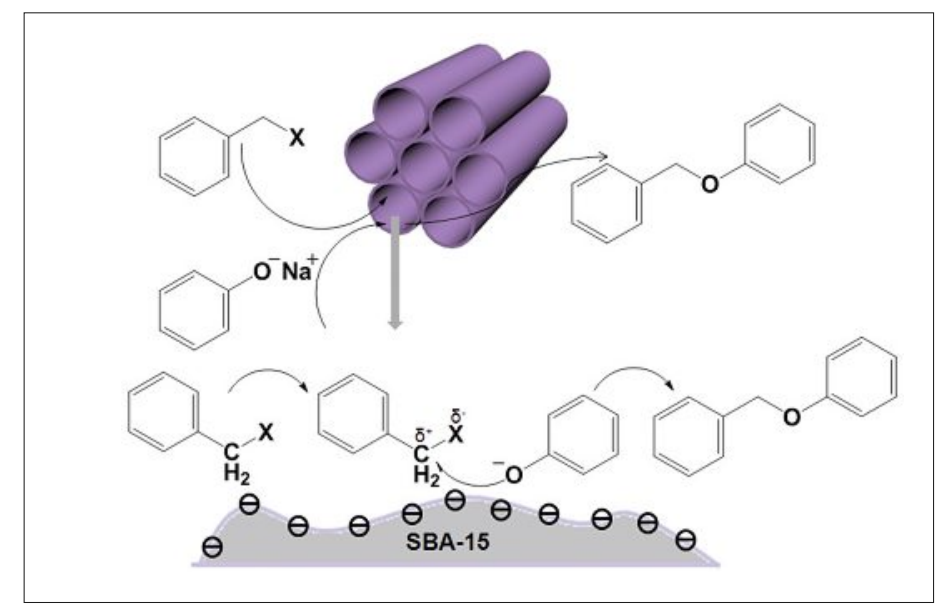

Fig. 1. The conceptual diagram showing the nucleophilic reaction in the SBA-15 nanoreactors.
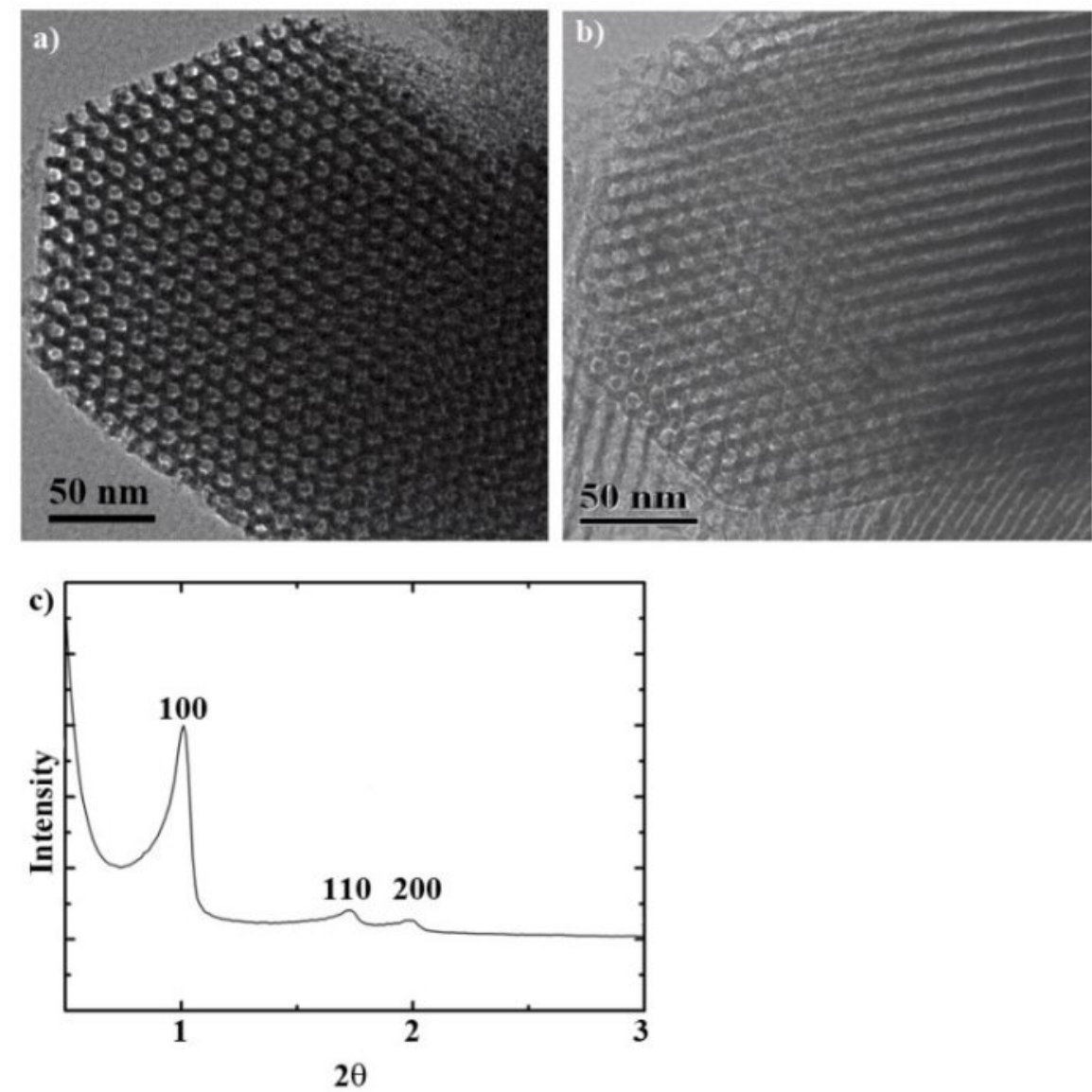

Fig. 2. TEM images of mesoporous silica SBA-15 along the [001] direction a) before and b) after reaction. c) XRD patterns of SBA-15. 
The accessibility of the two immiscible reactants to the solid catalyst is a key factor for the present reaction process. Indeed, the mesochannels of SBA-15 are amphiphilic due to the abundant hydrophilic $\mathrm{Si}-\mathrm{OH}$ groups and hydrophobic $\mathrm{Si}-\mathrm{O}-\mathrm{Si}$ groups on the silica surface. The SBA-15 materials are observed to be at the interface between the organic and the aqueous phases to catalyze the biphasic reactions. Consequently, the nanomaterial can host both hydrophobic benzyl halide and hydrophilic phenoxide within the pores, where the nucleophilic substitution can occur. This result is quite similar to the 'triphasic catalysis' reaction reported by Zhao and co-workers ${ }^{[11]}$ in which SBA-15$\mathrm{WO}^{4-}$ was used as a solid phase transfer reagent. To further confirm the importance of the amphiphilic properties, SBA-15 surface was modified with hydrophobic methyl groups (SBA-15- $\mathrm{CH}_{3}$ ) by using methyltriethoxysilane. ${ }^{[11]}$ With the surface modification, a remarkable decrease in the catalytic reaction rate was observed for SBA-15- $\mathrm{CH}_{3}$ in Fig. 3, implying that the more hydrophobic and uncharged environment is unfavorable for the reaction. The result indicates that less phenoxide can be loaded into the hydrophobic mesochannels thereby reducing the conversion rate.

Clearly as shown in Fig. 3, the nucleophilic substitution reaction rate with SBA-15 is at least as fast as that with $\mathrm{TBABr}$ and the complete conversion to benzylphenyl ether is obtained within two hours. The good performance of SBA-15 as a catalyst may be attributed to two important factors. First, the submicrometer structure of SBA-15 plays a significant role in improving the reaction efficiency. Due to the high amphiphilic surface area and the appropriate pore size, reactants (or products) can easily move into (or out of) SBA-15 mesochannels and have more interactions on the surface to react quickly. To test whether the pore size can affect the reaction rate, mesoporous MCM-41 with a smaller pore size $(2.5 \mathrm{~nm})^{[17]}$ was also used. Although MCM-41 possesses higher surface area, Fig. 3 shows that the corresponding nucleophilic reaction proceeds slightly slower as mass transfer into the pores is hindered, indicating that the reaction occurs inside the pores of SBA-15 and not only on the outer surface of the silica.

To study the influence of the surface charge, the zeta potential of SBA-15 was measured to be negative $(-12.8 \mathrm{mV})$. As shown in Fig. 1, when benzyl halide is close to the surface of SBA-15 via interaction with hydrophobic Si-O-Si groups, the negatively charged surface could also further polarize the $\mathrm{CH}_{2}-\mathrm{X}$ bond, making it easier for the benzylic carbon atom to be attacked by phenoxide. Consequently, the nucleophilic reaction is enhanced. To test this hypothesis, the SBA-15 was surfacemodified with amino functional groups and tested as catalyst (SBA-15-NH $)$. Fig. 3 illustrates that the rate of conversion to benzyl phenyl ether is lower with SBA$15-\mathrm{NH}_{2}$ than with SBA-15, for a given reaction time. When functionalized with amino groups, the zeta potential of SBA15 increases to $10.9 \mathrm{mV}$ and the surface becomes positively charged, thus resulting a slower nucleophilic reaction rate.

Given the general nature of nucleophilic substitutions, the catalysis can be extended to similar two-phase reactions. Some other examples are presented in Table 1 by using SBA-15 or TBABr as the catalyst for comparison. As observed, a higher percent conversion to ether can be achieved in the SBA15 nanoreactor. Compared with traditional PTCs, the SBA-15 catalyst is much more cost effective and environmental friendly. It is easy to recover the catalyst for facilitating the post-reaction processing. Moreover, the SBA-15 catalyst was easily recovered by filtration and washing with ethanol for removal of the organic phase. The TEM of the regenerated material was shown in Fig. 2b, and the structures were well preserved, which indicated the fine circulation. The regenerated material shows decrease in catalytic capacity down to $\sim 68 \%$ after the fifth regeneration. The decreases may be due to loss of the catalysts with washing.
Overall, this material shows good regeneration properties.

\section{Conclusions}

In summary, we demonstrated that liquid-liquid nucleophilic substitutions can be effectively carried out in the nanochannels of unmodified mesoporous silica (SBA-15) without phase transfer catalyst. The amphiphilic SBA-15, acting as a 'solid interfacial reactor', can host both hydrophilic and hydrophobic species in the mesochannels where a highly efficient nanoreaction can occur rapidly due to the confinement effect. High product conversion and reaction rates are achieved with a low catalyst loading for the synthesis of benzyl phenyl ether, which can be attributed to the enrichment effect of the mesochannels, the rapid diffusion and mass transfer with the appropriate submicrometer pores, and the further polarization of substrate molecules induced by the negatively charged SBA-15 surface for easier $S_{v} 2$ reaction. This 'green' method is economical compared to the expensive use of PTCs. Moreover, the product purification process is also simple as the separation of PTCs is not required. It is expected that the mesoporous silica materials would have potential industrial applications in other two-phase nucleophilic reactions.

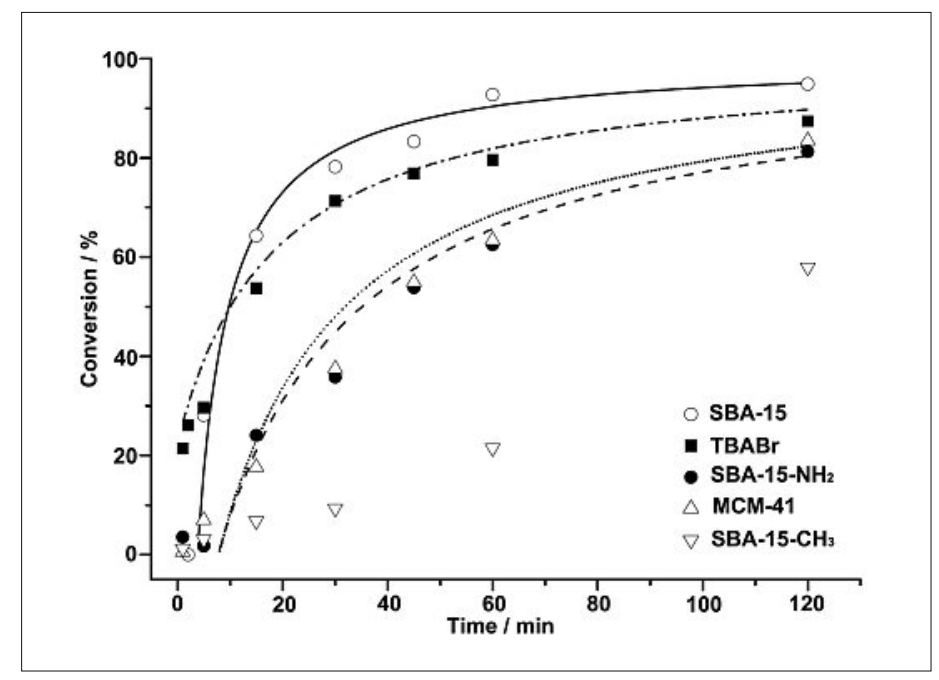

Fig. 3. Reaction profiles of the experimental data with fitted second-order kinetics reaction between benzyl bromide $(5 \mathrm{mmol})$ and $1.0 \mathrm{~mL}$ of aqueous phenoxide solution $(10.0$ $\mathrm{mmol}$ of $\mathrm{NaOH}$ and $7.5 \mathrm{mmol}$ of phenol). The reactions were carried out either with tetrabutylammonium bromide (TBABr, 0.15 $\mathrm{mmol}$ ) or different mesoporous silica materials as noted (2 mg).

Table 1. The nucleophilic substitution reaction catalyzed by SBA-15 or TBABr.

\begin{tabular}{|c|c|c|c|c|c|}
\hline Entry & $\mathrm{PhCH}_{2} \mathrm{X}$ & $\mathrm{PhOH}$ & Time $[\mathrm{min}]$ & $\mathrm{T}\left[{ }^{\circ} \mathrm{C}\right]$ & Conversion $[\%]^{\mathrm{c}}$ \\
\hline $1^{\mathrm{a}}$ & $\mathrm{X}=\mathrm{Cl}$ & $\mathrm{C}_{6} \mathrm{H}_{5} \mathrm{OH}$ & 240 & 60 & 94 \\
\hline $2^{\mathrm{b}}$ & $\mathrm{X}=\mathrm{Cl}$ & $\mathrm{C}_{6} \mathrm{H}_{5} \mathrm{OH}$ & 240 & 60 & 89 \\
\hline $3^{\mathrm{a}}$ & $\mathrm{X}=\mathrm{Br}$ & $p$-Cresol & 300 & 75 & 45 \\
\hline $4^{\mathrm{b}}$ & $\mathrm{X}=\mathrm{Br}$ & $p$-Cresol & 300 & 75 & 39 \\
\hline
\end{tabular}

The two-phase reaction is catalysed by aSBA-15 or ${ }^{\mathrm{b}} \mathrm{TBABr}$. ${ }^{\mathrm{S}}$ Products were determined by HPLC. 


\section{Acknowledgements}

This work was supported by NSFC (20925517, 21175028, 21105014), STCSM (10XD1406000), SKLEAC201101, and Texas State University Faculty Development Leave Program.

Received: May 11, 2018

[1] C. M. Starks, C. L. Liotta, M. Halpern, 'Phase Transfer Catalysis: Fundamental, Applications and Industrial Perspectives', Chapman \& Hall New York, 1994

[2] F. M. Menger, A. R. Erlington, J. Am. Chem. Soc. 1991, 113, 9621.

[3] A. Nelson, Angew. Chem. Int. Ed. 1999, 38, 1583

[4] R. Annunziata, M. Benaglia, F. Cozzi, G. Tocco, Org. Lett. 2000, 2, 1737.

[5] S. Gmouh, J. Jammal-Eddine, J. Y. Valnot, Tetrahedron 2000, 56, 8361.

[6] N. M. T. Lourenco, C. A. M. Afonso, Tetrahedron 2003, 59, 789 .
[7] M. Poliakoff, J. M. Fitzpatrick, T. R. Farren, P. T. Anatas, Science 2002, 297, 807

[8] S. Park, Curr. Appl. Phys. 2008, 8, 664.

[9] C. Wheeler, K. N. West, C. L. Liotta, C. A. Eckert, Chem. Commun. 2001, 10, 887.

[10] a) A. Corma, Chem. Rev. 1997, 97, 2373; b) C. Li, Catal. Rev. 2004, 46, 419; c) X. S. Zhao, X. Y. Bao, W. P. Guo, F. Y. Lee, Mater. Today 2006, 9, 32; d) C. J. Liu, S. J. Li, W. Q. Pang, C. M. Che, Chem. Commun. 1997, 1, 65; e) K. Qian, J. Wan, F. Liu, H. Girault, B. Liu, C. Yu, ACS nano 2009, 11, 3656; f) X. Sun, L. Huang, R. Zhang, W. Xu, J. Huang, D. Gurav, V. Vedarethinam, R. Chen, J. Lou, Q. Wang, J. Wan, K. Wan, ACS Cent. Sci. 2018, 4, 223; g) L. Huang, J. Wan, X. Wei, J. Huang, R. Zhang, D. Gurav, V. Vedarethinam, Y. Li, R. Chen, K. Qian, Nat. Commun. 2017, 8, 220; h) X. Sun, J. Wan, K. qian, Small Meth. 2017, 1, 1700196.

[11] a) R. Y. Zhang, W. Ding, B. Tu, D. Y. Zhao, Chem. Mater. 2007, 19, 4379; b) A. Bhaumik, P. Mukherjee, R. Kumar, J. Catal. 1998, 178, 101; c) R. Deam, A. Dayal, T. McAllister, A. Mundy, R. Western, L. Besley, A. Farmer, E. Horrigan,
A. Murphy, J. Chem. Soc. Chem.Commun. $1995,349$.

[12] a) S. L. Regen, J. Am. Chem. Soc. 1975, 97 , 5956; b) M. L. Wang, Z. F. Lee, F. S. Wang, Ind Eng. Chem. Res. 2005, 44, 5417.

[13] a) H. Zhang, J. Sun, D. Ma, G. Weinberg, D. S Su, X. Bao, J. Phys. Chem. B 2006, 110, 25908; b) H. Zhang, J. Sun, D. Ma, X. Bao, A. KleinHoffmann, G. Weinberg, D. S. Su, R. Scholgl, $J$. Am. Chem. Soc. 2004, 126, 7440; c) B. C. Chen, H. P. Lin, M. C. Chao, C. Y. Mou, C. Y. Tang, Adv. Mater. 2004, 16, 1657.

[14] N. R. Shiju, A. H. Alberts, S. Khalid, D. R Brown, G. Rothenberg, Angew. Chem. Int. Ed. 2011, 41, 9615 .

[15] S. France, M. H. Shah, A. Weatherwax, H. Wack, J. P. Roth, T. Lectka, J. Am. Chem. Soc. 2005, 127, 1206

[16] D. Y. Zhao, J. L. Feng, Q. S. Huo, N. Melosh, G. H. Fredrickson, B. F. Chmelka, G. D. Stucky, Science 1998, 279, 548.

[17] K. Sato, M. Aoki, J. Takagi, R. Noyori, J. Am. Chem. Soc. 1997, 119, 12386. 